\section{Stillverhalten von Frauen mit rheumatoider Arthritis}

Ince-Askan $\mathrm{H}$ et al. Breastfeeding among Women with Rheumatoid Arthritis Compared with the General Population: Results from a Nationwide Prospective Cohort Study. J Rheumatol 2019; 46: 1067-1074

Die Weltgesundheitsorganisation empfiehlt, dass Säuglinge bis zum Alter von 6 Monaten ausschließlich gestillt werden. Im Rahmen der prospektiven PARA-Studie (Pregnancy-induced Amelioration of Rheumatoid Arthritis) wurde die Häufigkeit des Stillens und der Zeitpunkt der Beendigung zwischen Frauen mit rheumatoider Arthritis (RA) und der Allgemeinbevölkerung verglichen und Gründen gesucht, warum RA-Patientinnen mit dem Stillen aufhören.

Von 2002 bis 2008 wurden insgesamt 216 Frauen von der Schwangerschaft bis $6 \mathrm{Mo}-$ nate nach der Geburt überprüft (insgesamt 249 Schwangerschaften). Es wurden Daten zur Stillzeit und zum Medikamentengebrauch gesammelt. Verglichen wurde der prozentuale Anteil des Stillens in der Studienpopulation mit dem der vergleichbarer Personengruppen in der Allgemeinbevölkerung. Das mittlere Alter der Studienteilnehmer zum Zeitpunkt der Geburt lag bei 32,8 Jahren und die Dauer der rheumatoiden Arthritis betrug durchschnittlich 4,9 jahre.

Die Gelenkfunktion und damit das Ausmaß einer eingeschränkten Beweglichkeit der RA-Patientinnen wurde mit dem HAQScore (Healthcare Access and Quality) beurteilt. Einen HAQ-Score $<0,75$ hatten 45,8\% der Patientinnen und 54,2\% einen HAQ-Score $\geq 0,75$ Insgesamt wurden 74,7\% der Patientinnen $(n=186)$ als Rheumafaktor (RF) positiv oder ACPA positiv (Antikörper gegen citrullinierte Proteine) klassifiziert. Etwa die Hälfte der Patientinnen hatte einen hohen sozioökonomischen Status und 22 rauchten während der Schwangerschaft.

Insgesamt $43 \%$ der Patientinnen stillten ihr Neugeborenes mindestens 4-6 Wochen, $26 \%$ bis zur 12 Woche und $9 \%$ bis zur 26 . Woche nach der Geburt. Die Werte in der Referenzbevölkerung lagen bei 63, 46 und $41 \%$. Der Unterschied war signifikant. Still- frequenz und Dauer unterschieden sich nicht zwischen Nulliparae und Multiparae in der PARA-Studiengruppe.

Der Hauptgrund für Frauen, das Stillen abzubrechen, war die Wiederaufnahme der Medikation. Insgesamt nahmen 58,9\% der RA-Patientinnen, die das Stillen wegen der Wiederaufnahme der Medikation abbrachen, Arzneimittel ein, die mit dem Stillen nicht vereinbar waren, aber mehr als $40 \%$ dieser Patientinnen nahmen als stillverträglich geltenden Medikamente ein. Insgesamt gaben 42,2\% der Patientinnen andere Gründe als die Medikamenteneinnahme an, das Stillen abzubrechen. Die mütterlichen Hauptgründe waren dabei „nicht genügend Milch“ und „kein Verlangen“. Gründe, die mit dem Neugeborenen zusammenhingen waren „sperren des Kindes“ und „fehlendes Gedeihen“. Diese aufgeführten Gründe standen dabei im Einklang mit denen der Referenzbevölkerung.

\section{FAZIT}

Die Ergebnisse dieser großen prospektiven Studie zeigen, dass RA-Mütter ihre Kinder zu einem geringeren Anteil stillen und das Stillen früher abbrechen, als Frauen in der Allgemeinbevölkerung. Eine beträchtliche Anzahl der Studienteilnehmerinnen brach das Stillen ab, um mit der Einnahme von Medikamenten zu beginnen, obwohl viele der Medikamente als sicher in der Stillzeit eingestuft werden. Die Autoren empfehlen anhand der Studienergebnisse Interventionsstrategien zur Unterstützung von RA-Patientinnen, die stillen möchten.

\section{Richard Kessing, Zeiskam}

